\title{
Universal quantum entanglement between an oscillator and continuous fields
}

\author{
Haixing Miao, ${ }^{1}$ Stefan Danilishin, ${ }^{2,3}$ and Yanbei Chen ${ }^{4}$ \\ ${ }^{1}$ School of Physics, University of Western Australia, Western Australia 6009, Australia \\ ${ }^{2}$ Physics Faculty, Moscow State University, Moscow 119991, Russia \\ ${ }^{3}$ Max-Planck Institut für Gravitationsphysik (Albert-Einstein-Institut) and Leibniz Universität Hannover, \\ Callinstr. 38, D-30167 Hannover, Germany \\ ${ }^{4}$ Theoretical Astrophysics 130-33, California Institute of Technology, Pasadena, California 91125, USA
}

(Received 7 August 2009; published 7 May 2010)

\begin{abstract}
Quantum entanglement has been actively sought in optomechanical and electromechanical systems. The simplest system is a mechanical oscillator interacting with a coherent optical field, while the oscillator also suffers from thermal decoherence. With a rigorous functional analysis, we develop a mathematical framework for treating quantum entanglement that involves infinite degrees of freedom. We show that the quantum entanglement is always present between the oscillator and continuous optical field—even when the environmental temperature is high and the oscillator is highly classical. Such a universal entanglement is also shown to be able to survive more than one mechanical oscillation period if the characteristic frequency of the optomechanical interaction is larger than that of the thermal noise. In addition, we introduce effective optical modes that are ordered by the entanglement strength to better understand the entanglement structure, analogously to the energy spectrum of an atomic system. In particular, we derive the optical mode that is maximally entangled with the mechanical oscillator, which will be useful for future quantum computing and encoding information into mechanical degrees of freedom.
\end{abstract}

DOI: 10.1103/PhysRevA.81.052307

PACS number(s): 03.67.Bg, 03.65.Ta, 03.67.Mn, 42.50.Wk

\section{INTRODUCTION}

Entanglement, as one of the most fascinating features of quantum mechanics, lies in the heart of quantum computing and many quantum communication protocols [1]. Great efforts have been devoted to theoretical and experimental investigations of quantum entanglement in different systems with discrete or continuous variables. Due to recent significant achievements in fabricating high- $Q$ mechanical oscillators, quantum entanglement with mechanical degrees of freedom has aroused great interest. In particular, many table-top experiments have demonstrated significant cooling of mechanical degrees of freedom via feedback or passive damping (self-cooling) [2-22], which in principle allows us to achieve the quantum ground state [23-28]. More recently, with a conventional cryogenic refrigeration, O'Connell et al. has succeeded in the ground-state cooling of a micromechanical oscillator [29]. These experiments not only illuminate quantum-limited measurements [30] but also pave the way for creating quantum entanglement with mechanical degrees of freedom. Theoretical analysis shows that by coupling a mechanical oscillator to a Fabry-Perot cavity, one can create stationary (Einstein-Podolsky-Rosen [EPR]) EPR-type quantum entanglement between optical modes and an oscillator [31-33] or even between two macroscopic oscillators [34,35]. In Ref. [36], it was shown that entanglement between two oscillators can also be created by conditioning on continuous measurements of the common and differential optical modes in a laser interferometer.

Here we consider the quantum entanglement between a mechanical oscillator and a coherent optical field, which models the essential process in all above-mentioned optomechanical systems. There are two important motivations behind this. The first is in regard to evaluation of entanglement involving a field which contains a field of infinite degrees of freedom.
The entanglement structure itself is an interesting problem. To our knowledge, only finite-degrees-of-freedom entanglements have been investigated in the literature. The second reason in regard to the effect of thermal decoherence. There is an interesting observation: on the one side, the environmental temperature enters as an explicit factor and directly determines the existence of the optomechanical entanglement considered in Refs. [31,34,35]; on the other side, only the ratio between the optomechanical interaction and thermal decoherence determines the existence of the entanglement instead of the thermal decoherence alone, and the environmental temperature influences only the entanglement strength implicitly as shown in Refs. [36,37]. By studying this essential process, we can have a complete picture of the thermal decoherence.

The model and its space-time diagram are shown schematically in Fig. 1. A similar system was analyzed previously by Pirandola et al. [38]. They used a narrow-detection-band approximation to introduce sideband modes, which maps the outgoing field into two effective degrees of freedom. In the situation here, sideband modes are not well defined, because the interaction turns off at $t=0$ and only half-space $[-\infty, 0]$ is involved. Instead, we will directly evaluate the entanglement between the oscillator and outgoing field $\hat{b}$ (infinite degrees of freedom) using the positivity of partial transpose (PPT) criterion [39-46]. Only in the weak-interaction and lowthermal-noise limit can we make correspondences between our results and those obtained in Ref. [38].

The outline of this article is as follows: In Sec. II, we will analyze the dynamics of this system and introduce the covariance between the dynamical quantities, which will be essential for analyzing the quantum entanglement. In Sec. III, we take the continuous limit and extend the PPT criterion to the case with infinite degrees of freedom. In addition, we apply a rigorous functional analysis and obtain the entanglement measure of which a simple scaling is derived. In Sec. IV, we study the 

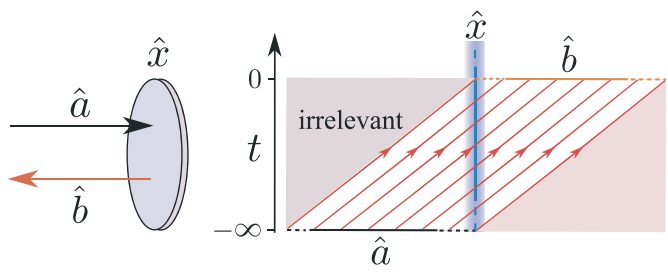

FIG. 1. (Color online) A schematic plot of the model and the corresponding space-time diagram. Here $\hat{x}, \hat{a}$, and $\hat{b}$ denote the oscillator position and ingoing and outgoing fields, respectively. For clarity, we intentionally place $\hat{a}$ and $\hat{b}$ on difference sides of the oscillator world line. The tilted lines represent the light rays. Up to some instant we are concerned with $(t=0)$, the optical field entering later is out of causal contact and thus irrelevant.

survival time of the entanglement under thermal decoherence for such entanglement. In Sec. V, we introduce effective optical modes to understand the entanglement structure and obtain the maximally entangled mode. In Sec. VI, we make a numerical estimate given a set of experimental achievable specification to motivate future experiment to investigate the entanglement. We conclude our main results in Sec. VII.

\section{DYNAMICS AND COVARIANCE MATRIX}

Due to the linearity of system dynamics, the Heisenberg equations of motion are formally identical to the classical equations of motion, apart from the fact that every dynamical quantity is now treated as a quantum operator. For the optical field, the standard input-output relation reads as follows:

$$
\begin{gathered}
\hat{b}_{1}(t)=\hat{a}_{1}(t), \\
\hat{b}_{2}(t)=\hat{a}_{2}(t)+\kappa \hat{x}(t) .
\end{gathered}
$$

Here $\hat{a}_{1}\left(\hat{b}_{1}\right)$ and $\hat{a}_{2}\left(\hat{b}_{2}\right)$ are amplitude and phase quadratures of the ingoing (outgoing) optical field. They are defined by the optical electric field: $\hat{E}_{\mathrm{in}}(t)=\sqrt{\frac{4 \pi \hbar \omega_{0}}{\mathcal{S} c}}\left[\left(\bar{a}+\hat{a}_{1}(t)\right) \cos \omega_{0} t+\right.$ $\left.\hat{a}_{2}(t) \sin \omega_{0} t\right]$, which contains a steady-state part $\bar{a}$ and quantum fluctuation parts $\hat{a}_{1,2}$. In this equation, $\mathcal{S}$ is the transverse sectional area of the optical beam, $\bar{a}=\sqrt{I_{0} /\left(\hbar \omega_{0}\right)}$ with $I_{0}$ the optical power, and $\omega_{0}$ is the laser frequency. A similar relation for $\hat{E}_{\text {out }}$ and $\hat{b}_{1,2}$ also holds. In Eq. (2), the displacement of the mechanical oscillator $\hat{x}$ modulates the phase quadrature of the outgoing optical field with an optomechanical coupling constant $\kappa \equiv \omega_{0} \bar{a} / c$. To quantify the interaction strength, we introduce a characteristic interaction frequency $\Omega_{q}$ which is defined through $\Omega_{q} \equiv \sqrt{\hbar \kappa^{2} / m}$.

For the mechanical oscillator, the equations of motion are given by

$$
\begin{gathered}
\dot{\hat{x}}(t)=\frac{\hat{p}(t)}{m}, \\
\dot{\hat{p}}(t)=-\gamma_{m} \hat{p}(t)-m \omega_{m}^{2} \hat{x}(t)+\hat{F}_{\mathrm{rad}}(t)+\hat{\xi}_{\mathrm{th}}(t) .
\end{gathered}
$$

Here $\hat{p}$ is the oscillator momentum. To include the fluctuationdissipation mechanism of the oscillator coupled to the thermal heat bath at temperature $T$, we have introduced the mechanical damping $\gamma_{m}$ and the corresponding thermal force noise $\hat{\xi}_{\text {th }}$ which has the following correlation function in the high-temperature limit: $\left\langle\hat{\xi}_{\text {th }}(t) \xi_{\text {th }}\left(t^{\prime}\right)\right\rangle=2 m \gamma_{m} k_{B} T \delta\left(t-t^{\prime}\right) \equiv$
$2 \hbar m \Omega_{F}^{2} \delta\left(t-t^{\prime}\right)$ with $\Omega_{F}$ a characteristic frequency of the thermal noise. The presence of thermal noise $\hat{\xi}_{\text {th }}$ ensures the correct commutator between $\hat{x}(t)$ and $\hat{p}(t)$ [47]. The radiationpressure force $\hat{F}_{\text {rad }}$, up to the first order in the quantum fluctuation, is proportional to $\hat{a}_{1}(t)$ and $\hat{F}_{\text {rad }}(t)=\hbar \kappa \hat{a}_{1}(t)$.

The above equations completely quantify the linear dynamics of the system and they can be easily solved. The solution to oscillator position $\hat{x}$ is simply

$$
\hat{x}(t)=\int_{-\infty}^{t} d t^{\prime} G_{x}\left(t-t^{\prime}\right)\left[\hbar \kappa \hat{a}_{1}\left(t^{\prime}\right)+\hat{\xi}_{\mathrm{th}}\left(t^{\prime}\right)\right],
$$

where Green's function $G_{x}(t) \equiv e^{-\gamma_{m} t} \sin \left(\omega_{m} t\right) /\left(m \omega_{m}\right)$. The radiation-pressure term $\hbar \kappa \hat{a}_{1}$ induces quantum correlations between the oscillator and the optical field, but it is undermined by $\hat{\xi}_{\text {th }}$. The question would be whether quantum entanglement exists after evolving the entire system from $t=-\infty$ to 0 .

Since variables involved are Gaussian and linear dynamics will preserve Gaussian conditions, the quantum entanglement is completely encoded in the covariance matrix $\mathbf{V}$. With the optical field labeled by continuous coordinate $t$, elements of $\mathbf{V}$ involving optical degrees of freedom would be defined in the functional space $\mathcal{L}^{2}[-\infty, 0]$. Specifically,

$$
\mathbf{V}=\left[\begin{array}{ll}
\mathbf{A} & \mathbf{C}^{\mathbf{T}} \\
\mathbf{C} & \mathbf{B}
\end{array}\right]
$$

Here $\quad \mathbf{A}_{i j}=\left\langle\vec{X}_{i} \vec{X}_{j}\right\rangle_{\text {sym }}(i, j=1,2) \quad$ with $\quad$ vector $\quad \vec{X} \equiv$ $[\hat{x}(0), \hat{p}(0)]$ and $\left\langle\vec{X}_{i} \vec{X}_{j}\right\rangle_{\text {sym }} \equiv\left\langle\vec{X}_{i} \vec{X}_{j}+\vec{X}_{j} \vec{X}_{i}\right\rangle / 2$ denoting symmetrized ensemble average; $\mathbf{C}_{i j}$ and $\mathbf{B}_{i j}$ should be viewed as vectors and operators in $\mathcal{L}^{2}[-\infty, 0]$. In the coordinate representation, $\left(t \mid \mathbf{C}_{i j}\right)=\left\langle\vec{X}_{i} \hat{b}_{j}(t)\right\rangle_{\mathrm{sym}}$ and $\left(t\left|\mathbf{B}_{i j}\right| t^{\prime}\right)=\left\langle\hat{b}_{i}(t) \hat{b}_{j}\left(t^{\prime}\right)\right\rangle_{\text {sym }}$, where $(\mid)$ denotes the scalar inner product in $\mathcal{L}^{2}[-\infty, 0]$.

\section{UNIVERSAL ENTANGLEMENT}

According to Refs. $[43,46]$, in order for one particle and a joint system of arbitrarily large $N$ particles to be separable, a necessary and sufficient condition is that partially transposed density matrix $\varrho_{1 \mid N}^{\mathbf{T}_{1}}$ (with respect to the first particle) should be positive semidefinite, i.e., $\varrho_{1 \mid N}^{\mathbf{T}_{1}} \geqslant 0$. In the phase space of continuous Gaussian variables, this reduces to the uncertainty principle,

$$
\mathbf{V}_{\mathrm{pt}}+\frac{1}{2} \mathbf{K} \geqslant 0 \text {. }
$$

Here the commutator matrix $\mathbf{K}=\bigoplus_{k=1}^{N+1} 2 \sigma_{y}$, with $\sigma_{y}$ denoting the Pauli matrix. According to the Williamson theorem, there exists a symplectic transformation $\mathbf{S} \in S_{p(2 N+2, \mathbb{R})}$ such that $\mathbf{S}^{\mathrm{T}} \mathbf{V}_{\mathrm{pt}} \mathbf{S}=\bigoplus_{k=1}^{N+1} \operatorname{Diag}\left[\lambda_{k}, \lambda_{k}\right]$. Using the fact that $\mathbf{S}^{\mathrm{T}} \mathbf{K} \mathbf{S}=\mathbf{K}$, the above uncertainty principle reads $\lambda_{k} \geqslant 1$. If this fails to be the case, i.e., $\exists \lambda_{k}<1$, the states are entangled. The amount of entanglement can be quantified by the logarithmic negativity $E_{\mathcal{N}}$ [44], which is defined as

$$
E_{\mathcal{N}} \equiv \max \left[-\sum_{k} \ln \lambda_{k}, 0\right] \quad \text { for } \quad k: \lambda_{k}<1 .
$$

In the case considered here, $N$ approaches $\infty$ and the partial transpose is equivalent to time reversal and therefore 
$\mathbf{V}_{\mathrm{pt}}=\left.\mathbf{V}\right|_{\hat{p}(0) \rightarrow-\hat{p}(0)}$. According to Ref. [44], $\lambda_{k}$ can be obtained by solving an eigenvalue problem:

$$
\mathbf{V}_{\mathrm{pt}} \mathbf{v}=\frac{1}{2} \lambda \mathbf{K v},
$$

where $\left.\left.\mathbf{v} \equiv\left[\alpha_{0}, \beta_{0}, \mid \alpha\right), \mid \beta\right)\right]^{\mathbf{T}}$ with $\left.\mid f\right)$ denoting the vector in $\mathcal{L}^{2}[-\infty, 0]$. Normalizing $\hat{x}$ and $\hat{p}$ with respect to their zero-point values, the commutator reads $[\hat{x}, \hat{p}]=2 i$. For the optical field, we set $\left[\hat{b}_{1}(t), \hat{b}_{2}\left(t^{\prime}\right)\right]=2 i \delta\left(t-t^{\prime}\right)$, which gives the coordinate representation of $\mathbf{K}$.

Due to uniqueness of $\mid \alpha)$ and $\mid \beta)$ in terms of $\alpha_{0}$ and $\beta_{0}$ for any $\lambda<1$ (nonsingular), Eq. (9) leads to the following characteristic equation [cf. Eq. (6)]

$$
\operatorname{det}\left[\mathbf{A}+\lambda \sigma_{y}-\mathbf{C}^{\mathbf{T}}\left(\lambda \sigma_{y}+\mathbf{B}\right)^{-1} \mathbf{C}\right]=0 .
$$

It can be shown that

$$
\left(\lambda \sigma_{y}+\mathbf{B}\right)^{-1}=\left[\begin{array}{cc}
1+B_{\lambda}^{\dagger} M^{-1} B_{\lambda} & -B_{\lambda}^{\dagger} M^{-1} \\
-M^{-1} B_{\lambda} & M^{-1}
\end{array}\right],
$$

where we have used the fact that $B_{12}^{\dagger}=B_{21}$ in $\mathcal{L}^{2}[-\infty, 0]$ and have defined $B_{\lambda} \equiv B_{12}-i \lambda$ and $M \equiv B_{22}-B_{\lambda}^{\dagger} B_{\lambda}$.

To solve this characteristic equation, we need to invert operator $M$ which can be achieved via the Wiener-Hopf method. ${ }^{1}$ Given any function $\left.\left.\mid g\right)=M^{-1} \mid h\right)$, in the frequency domain, it reads

$$
\left.\tilde{g}(\Omega)=\int_{-\infty}^{0} d t e^{i \Omega t} M^{-1} \mid h\right)=\frac{1}{\tilde{\psi}_{-}}\left[\frac{\tilde{h}}{\tilde{\psi}_{+}}\right]_{-} .
$$

Here [ ] means taking the causal part of given function (with poles in lower-half complex plane) and factorization

$$
\tilde{\psi}_{+} \tilde{\psi}_{-} \equiv \Lambda+i \lambda \hbar \kappa^{2}\left(\tilde{G}_{x}-\tilde{G}_{x}^{*}\right)+2 \hbar m \kappa^{2} \Omega_{F}^{2} \tilde{G}_{x} \tilde{G}_{x}^{*}
$$

with $\Lambda \equiv 1-\lambda^{2}$ and $\tilde{G}_{x}$ denoting the Fourier transformation of $G_{x}(t)$. In the above equation, $\tilde{\psi}_{+}\left(\tilde{\psi}_{-}\right)$and its inverse are analytic in upper-half (lower-half) complex plane, $\tilde{\psi}_{+}(-\Omega)=\tilde{\psi}_{+}^{*}(\Omega)=\tilde{\psi}_{-}(\Omega)$. In deriving Eq. (13), we have used $\left\langle\hat{a}_{i}(t) \hat{a}_{j}\left(t^{\prime}\right)\right\rangle_{\mathrm{sym}}=\delta_{i j} \delta\left(t-t^{\prime}\right)$, and the correlation function for the thermal noise.

Finally, an implicit polynomial equation for the simplectic eigenvalue $\lambda$ is derived from Eq. (10). As it turns out, there always exists one eigenvalue $\lambda$ that is smaller than 1 . In Fig. 2, the corresponding logarithmic negativity [cf. Eq. (8)] is shown as a function of $\Omega_{q} / \Omega_{F}$. For a high- $Q$ oscillator $Q_{m} \equiv \omega_{m} / \gamma_{m} \gg 1$, up to the leading order of $1 / Q_{m}$, a very elegant expression for $E_{\mathcal{N}}$ is derived and it is

$$
E_{\mathcal{N}}=\frac{1}{2} \ln \left[1+\frac{25}{8} \frac{\Omega_{q}^{2}}{\Omega_{F}^{2}}\right] .
$$

It depends only on the ratio between $\Omega_{q}$ and $\Omega_{F}$, which clearly indicates the universality of the quantum entanglement. The reason why thermal decoherence $\left(\Omega_{F}\right)$ alone determines the existence of entanglement in Refs. [31,34,35] originates from the finite transmission of the cavity, and the information of the cavity mode and the oscillator motion leaks into the

\footnotetext{
${ }^{1}$ An introduction of this method in solving a similar problem can be found in the appendix of Ref. [50].
}

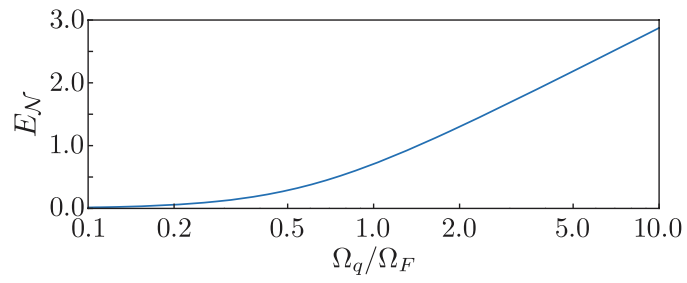

FIG. 2. (Color online) Logarithmic negativity $E_{\mathcal{N}}$ as a function of the ratio $\Omega_{q} / \Omega_{F}$. A mechanical quality factor $Q_{m}=10^{3}$ is chosen.

environment and induces additional decoherence. This is addressed thoroughly in Ref. [48].

\section{ENTANGLEMENT SURVIVAL DURATION}

To investigate how long such entanglement can survive under thermal decoherence, after turning off the optomechanical coupling at $t=0$, the mechanical oscillator freely evolves for a finite duration $\tau$, driven only by thermal noise. Due to thermal decoherence, entanglement will gradually vanish. Mathematically, the simplectic eigenvalue will become larger than unity when $\tau$ is larger than the survival time $\tau_{s}$. By replacing $[\hat{x}(0), \hat{p}(0)]$ with $[\hat{x}(\tau), \hat{p}(\tau)]$ and making similar analysis, up to the leading order of $1 / Q_{m}, \tau_{s}$ satisfies a transcendental equation:

$$
4 \Omega_{F}^{4} \theta_{s}^{2}-\left(2 \Omega_{F}^{2}+\Omega_{q}^{2}\right)^{2} \sin ^{2} \theta_{s}-25 \omega_{m}^{4}=0,
$$

with $\theta_{s} \equiv \omega_{m} \tau_{s}$. In the case of $\Omega_{q}<\Omega_{F}<\omega_{m}$, the oscillating term can be neglected, leading to

$$
\theta_{s}=\frac{5}{2} \frac{\omega_{m}^{2}}{\Omega_{F}^{2}}=\frac{5 Q_{m}}{2 \bar{n}_{\mathrm{th}}+1},
$$

where we have defined the thermal occupation number $\bar{n}_{\text {th }}$ through $k_{B} T /\left(\hbar \omega_{m}\right)=\bar{n}_{\text {th }}+(1 / 2)$. Therefore, in this case if $Q_{m}$ is larger than $\bar{n}_{\text {th }}$, the entanglement will be able to survive longer than one oscillation period. Since $Q_{m}>\bar{n}_{\text {th }}$ is also the requirement that the thermal noise induces an momentum diffusion smaller than its zero-point uncertainty [30], this condition is what we intuitively expect. In the strong interaction case with $\Omega_{q} \gg \Omega_{F}$, the transcendental equation can be solved numerically, showing that $\theta_{s}>1$ is always valid and the entanglement can survive at least up to one oscillation period.

\section{MAXIMALLY ENTANGLED MODE}

To gain insights into the structure of this entanglement, we apply the techniques in Ref. [49] and decompose outgoing field into independent single modes by convoluting them with some weight functions $f_{i}$, namely

$$
\hat{O}_{i} \equiv\left(f_{i} \mid \hat{b}\right),\left[\hat{O}_{i}, \hat{O}_{j}^{\dagger}\right]=2 \delta_{i j},
$$

which requires $\left(f_{i} \mid f_{j}\right)=\delta_{i j}$. If we define $g_{i 1} \equiv \operatorname{Re}\left[f_{i}\right]$ and $g_{i 2} \equiv \operatorname{Im}\left[f_{i}\right]$, the single-mode quadratures will be

$$
\begin{gathered}
\hat{X}_{i} \equiv\left(\hat{O}_{i}+\hat{O}_{i}^{\dagger}\right) / \sqrt{2}=\int_{-\infty}^{0} d t g_{i 1} \hat{b}_{1}-g_{i 2} \hat{b}_{2}, \\
\hat{Y}_{i} \equiv\left(\hat{O}_{i}-\hat{O}_{i}^{\dagger}\right) /(i \sqrt{2})=\int_{-\infty}^{0} d t g_{i 2} \hat{b}_{1}+g_{i 1} \hat{b}_{2} .
\end{gathered}
$$


Different choices of weight function will generally give optical modes that have different strength of entanglement with the mechanical oscillator. The function of particular interest is the one that gives an effective optical mode maximally entangled with the oscillator. Using the fact that logarithmic negativity is an entanglement monotone, the optimal weight function can be derived from the following constrained variational equation:

$$
\frac{\delta E_{\mathcal{N}}^{\mathrm{sub}}}{\delta g_{i}}+\mu_{i} g_{i}=0 \quad(i=1,2),
$$

where we have neglected unnecessary indices and $\mu_{k}$ is a Lagrange multiplier due to the constraint $(f \mid f)=1$ and $E_{\mathcal{N}}^{\text {sub }}$ quantifies entanglement in the subsystem consisting of the oscillator and the effective optical mode $[\hat{x}(0), \hat{p}(0), \hat{X}, \hat{Y}]$. As it turns out, the optimal weight functions $g_{1,2}$ have the shape of decay oscillation with poles $\omega$ given by the following polynomial equation

$$
\left[\left(\omega-\omega_{m}\right)^{2}+\gamma_{m}^{2}\right]\left[\left(\omega+\omega_{m}\right)^{2}+\gamma_{m}^{2}\right]+\chi=0,
$$

where parameter $\chi$ is a functional of $g_{1,2}$ and also depends on $\Omega_{q}$ and $\Omega_{F}$. Therefore, the weight functions are

$$
g_{k}(t)=A_{k} e^{\gamma_{g} t} \cos \left(\omega_{g} t+\theta_{k}\right) \quad(k=1,2),
$$

with $\gamma_{g}$ and $\omega_{g}$ being imaginary and real parts of $\omega$. Analytical solutions to parameters $A_{k}, \omega_{g}, \gamma_{g}$, and $\theta_{k}$ require exact expression of $\chi$ in terms of $g_{k}, \Omega_{q}$, and $\Omega_{F}$, which is rather complicated. Instead, we numerically optimize those parameters to maximize $E_{\mathcal{N}}^{\text {sub }}$.

Taking into account $(f \mid f)=1, A_{1}$ and $A_{2}$ can be reduced to a single parameter $\zeta$, which is defined through

$$
A_{k}^{2}=\frac{4 \gamma_{g}\left(\gamma_{g}^{2}+\omega_{g}^{2}\right) \cos ^{2}[\zeta+k(\pi / 2)]}{\gamma_{g}^{2}+\omega_{g}^{2}+\gamma_{g}^{2} \cos \left(2 \theta_{k}\right)+\gamma_{g} \omega_{g} \sin \left(2 \theta_{k}\right)} .
$$

From Eq. (21), $\omega_{g}^{2}-\gamma_{g}^{2}=\omega_{m}^{2}-\gamma_{m}^{2}$. In addition, a local unitary transformation (rotation and squeezing) will not change the simplectic eigenvalue. Without loss of generality, we can fix that $\theta_{1}=\pi / 2$ and $\theta_{2}=0$. Therefore, only two parameters, $\omega_{g}$ and $\zeta$, need to be optimized.

In the special case of the weak-interaction and low-thermalnoise limit $\left(\Omega_{q}, \Omega_{F} \ll \omega_{m}\right)$, the optimal $\zeta_{\text {opt }}$ is equal to $\pi / 4$, which indicates $A_{1} \approx A_{2}=2 \sqrt{\gamma_{m}}$ for a high- $Q$ oscillator. Besides, as shown in the upper panel of Fig. 3, the optimal $\omega_{g}^{\mathrm{opt}}=\omega_{m}$, leading to

$$
f(t)=2 \sqrt{\gamma_{m}} e^{\gamma_{m} t \pm i \omega_{m} t+\phi_{0}} .
$$

Therefore, the optimal weight function has the same shape as Stokes and anti-Stokes sideband modes. This is similar to what been obtained in Refs. [38,49]. However, due to causality, the weight function here is defined in $\mathcal{L}^{2}[-\infty, 0]$ rather than $\mathcal{L}^{2}[-\infty, \infty]$, which is essential for defining sideband modes.

In the case of strong interaction and high thermal noise $\left(\Omega_{q}, \Omega_{F}>\omega_{m}\right)$, the optimal $\omega_{g}$ deviates from $\omega_{m}$ and depends on $\Omega_{F}$ and $\Omega_{q}$, as shown in the lower panel of Fig. 3 . More generally, the optimal $\zeta_{\text {opt }}=\pi / 3$ and $\omega_{g}^{\text {opt }}$ can be
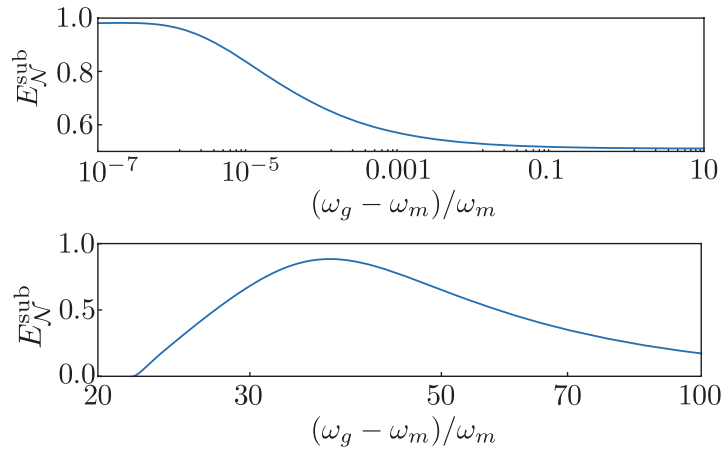

FIG. 3. (Color online) Logarithmic negativity $E_{\mathcal{N}}^{\text {sub }}$ as a function of quantity $\left(\omega_{g}-\omega_{m}\right) / \omega_{m}$ in the weak-interaction and low-thermalnoise case (upper panel) and strong-interaction and high-thermalnoise case (lower panel). In the first case, we have chosen $Q_{m}=$ $10^{3}, \Omega_{q} / \omega_{m}=\Omega_{F} / \omega_{m}=2 \times 10^{-2}$. In the second case, $Q_{m}=10^{6}$ (independent of $Q_{m}$ for higher $Q_{m}$ ), $\Omega_{q} / \omega_{m}=50, \Omega_{F} / \omega_{m}=20$, and $\zeta=\pi / 3$.

fitted by $\omega_{g}^{\text {opt }} \approx\left(0.64 \Omega_{F}^{2}+0.57 \Omega_{q}^{2}\right)^{1 / 2}$. Correspondingly, the logarithmic negativity can be approximated as

$$
E_{\mathcal{N}}^{\mathrm{sub}} \approx \frac{1}{2} \ln \left[1+\frac{15 . \Omega_{q}^{2}}{13 . \Omega_{F}^{2}+\Omega_{q}^{2}}\right]
$$

which again manifests universality of the entanglement. Therefore, as long as the optimal weight function is chosen, one can always recover quantum correlations between the oscillator and the outgoing field.

In principle, by choosing a weight function orthogonal to the optimal one obtained above, one can derive the nextorder optimal mode. Repeating this procedure will generate a complete spectrum of effective optical modes ordered by $E_{\mathcal{N}}^{\text {sub }}$, which is analogous to obtaining wave functions and corresponding energy levels with variational method in atomic systems. This not only helps in understanding the full entanglement structure but also sheds light on experimental verifications of such universal entanglement. Rather than trying to recover the infinite-dimension covariance matrix in Eq. (6), we can apply right weight functions to extract different effective optical modes and form low-dimension subsystems, for example, a subsystem consisting of the oscillator and the maximally entangled optical mode; more specifically, the corresponding $4 \times 4$ covariance matrix can be determined by measuring correlations among different quadratures. This can be achieved by using a local oscillator with time-dependent phase, which allows to probe both mechanical quadratures [50] and those of the effective optical mode. For example, a quadrature $\hat{O}_{\zeta}=\hat{X} \sin \zeta+\hat{Y} \cos \zeta$ can be measured with the following local oscillator light:

$$
L(t) \propto L_{1}(t) \cos \omega_{0} t+L_{2}(t) \sin \omega_{0} t
$$

with $\quad L_{1}(t)=g_{1}(t) \cos \zeta+g_{2}(t) \sin \zeta \quad$ and $\quad L_{2}(t)=$ $g_{2}(t) \cos \zeta-g_{1}(t) \sin \zeta$. Synthesis of multiple measurements will recover the covariance matrix that we need to verify the entanglement. 
TABLE I. Experimental specifications.

\begin{tabular}{ccccccc}
\hline \hline & $m$ & $\omega_{m} /(2 \pi)$ & $Q_{m}$ & $T$ & $I_{0}$ & $\eta$ \\
\hline Small scale & $50 \mathrm{ng}$ & $10^{5} \mathrm{~Hz}$ & $10^{7}$ & $4 \mathrm{~K}$ & $0.1 \mathrm{~W}$ & 0.05 \\
Large scale & $40 \mathrm{~kg}$ & $1 \mathrm{~Hz}$ & $10^{10}$ & $300 \mathrm{~K}$ & $800 \mathrm{~kW}$ & 0.05 \\
\hline \hline
\end{tabular}

\section{NUMERICAL ESTIMATE}

To motivate future experiments for realizing such a universal entanglement, we will include an important imperfection in a real experiment - the optical loss which comes from the finite transmission of the mirror. It will induce uncorrelated vacuum field $\hat{n}_{1,2}$ and the input-output relation will be modified into [cf. Eqs. (3) and (4)]

$$
\begin{gathered}
\hat{b}_{1}(t)=\sqrt{1-\eta} \hat{a}_{1}(t)+\sqrt{\eta} \hat{n}_{1}(t) \\
\hat{b}_{2}(t)=\sqrt{1-\eta}\left[\hat{a}_{2}(t)+\kappa \hat{x}(t)\right]+\sqrt{\eta} \hat{n}_{2}(t)
\end{gathered}
$$

with $\eta<1$ quantifying the optical loss. For a typical optical setup, $\eta$ can be the order of 0.05 or less. As it turns out, such a small optical loss almost has no effect on the entanglement strength.

To make numerical estimates and demonstrate experimental feasibility, we will consider experimentally achievable specifications for both small-scale and large-scale experiments, which are listed in Table I. For the small scale, it is chosen to be close to that of those table-top cooling experiments with micromechanical oscillator, and it gives $\Omega_{F} / \Omega_{q} \approx 40$ and $Q_{m} / \bar{n}_{\text {th }} \approx 10$. For the large scale, it is close to that of an advanced gravitational-wave detector with kg-scale test masses interacting with a high-power optical field [51], and we have $\Omega_{F} / \Omega_{q} \approx 1$ and $Q_{m} / \bar{n}_{\text {th }} \approx 10^{-3}$. In both cases, there is nonvanishing entanglement between the mechanical oscillator and the optical field, and the entanglement can survive up to one mechanical oscillation period.

\section{CONCLUSION}

We have demonstrated that quantum entanglement exists universally in system with a mechanical oscillator coupled to continuous optical field. The entanglement measurelogarithmic negativity displays an elegant scaling which depends on the ratio between characteristic interaction and thermal-noise frequency. Such scaling should also apply in electromechanical systems whose dynamics are similar to what we have considered.

\section{ACKNOWLEDGMENTS}

We thank F. Ya. Khalili, H. Müller-Ebhardt, H. Rehbein, K. Somiya, and our colleagues at the MQM group for invaluable discussions. The research of H. M. was supported by the Australian Research Council and the Department of Education, Science, and Training. S. D. was supported by the Alexander von Humboldt Foundation. Y. C. was supported by the Alexander von Humboldt Foundation's Sofja Kovalevskaja Program, NSF grants PHY-0653653 and PHY-0601459, as well as the David and Barbara Groce startup fund at Caltech.
[1] See e.g., D. Bouwmeester, A. Ekert, and A. Zeilinger, The Physics of Quantum Information (Springer, Berlin, 2002); S. L. Braunstein and P. Loock, Rev. Mod. Phys. 77, 513 (2005); R. Horodecki et al., ibid. 81, 865 (2009).

[2] D. G. Blair, E. N. Ivanov, M. E. Tobar, P. J. Turner, F. van Kann, and I. S. Heng, Phys. Rev. Lett. 74, 1908 (1995).

[3] P. F. Cohadon, A. Heidmann, and M. Pinard, Phys. Rev. Lett. 83, 3174 (1999).

[4] C. Höberger and K. Karrai, Nature 432, 1002 (2004).

[5] S. Gigan, H. R. Böhm, M. Paternostro, F. Blaser, G. Langer, J. B. Hertzberg, K. C. Schwab, D. Bäuerle, M. Aspelmeyer, and A. Zeilinger, Nature (London) 444, 67 (2006).

[6] O. Arcizet, P. Cohandon, T. Briant, M. Pinard, and A. Heidmann, Nature (London) 444, 71 (2006).

[7] D. Kleckner and D. Bouwmeester, Nature 444, 75 (2006).

[8] A. Schliesser, P. DelHaye, N. Nooshi, K. J. Vahala, and T. J. Kippenberg, Phys. Rev. Lett. 97, 243905 (2006).

[9] T. Corbitt, Y. Chen, E. Innerhofer, H. Muller-Ebhardt, D. Ottaway, H. Rehbein, D. Sigg, S. Whitcomb, C. Wipf, and N. Mavalvala, Phys. Rev. Lett. 98, 150802 (2007).

[10] T. Corbitt, C. Wipf, T. Bodiya, D. Ottaway, D. Sigg, N. Smith, S. Whitcomb, and N. Mavalvala, Phys. Rev. Lett. 99, 160801 (2007).

[11] A. Schliesser, R. Rivire, G. Anetsberger, O. Arcizet, and T. Kippenberg, Nature Physics 4, 415 (2008).
[12] M. Poggio, C. L. Degen, H. J. Mamin, and D. Rugar, Phys. Rev. Lett. 99, 017201 (2007).

[13] I. Favero, C. Metzger, S. Camerer, D. König, H. Lorenz, J. Kotthaus, and K. Karrai, Appl. Phys. Lett. 90, 104101 (2007).

[14] J. D. Teufel, J. W. Harlow, C. A. Regal, and K. W. Lehnert, Phys. Rev. Lett. 101, 197203 (2008).

[15] J. Thompson, B. Zwickl, A. Jayich, F. Marquardt, S. Girvin, and J. Harris, Nature 452, 72 (2008).

[16] C. M. Mow-Lowry, A. J. Mullavey, S. Goßler, M. B. Gray, and D. E. McClelland, Phys. Rev. Lett. 100, 010801 (2008).

[17] S. Gröblacher, S. Gigan, H. R. Böhm, A. Zeilinger, and M. Aspelmeyer, EPL 81, 54003 (2008).

[18] S. W. Schediwy, C. Zhao, L. Ju, D. G. Blair, and P. Willems, Phys. Rev. A 77, 013813 (2008).

[19] G. Jourdan, F. Comin, and J. Chevrier, Phys. Rev. Lett. 101, 133904 (2008).

[20] S. Gröblacher, J. Hertzberg, M. Vanner, G. Cole, S. Gigan, K. Schwab, and M. Aspelmeyer, Nature Physics 5, 485 (2009).

[21] B. Abbott et al. (LIGO Scientific Collaboration), New J. Phys. 11, 073032 (2009).

[22] T. Rocheleau, T. Ndukum, C. Macklin, J. Hertzberg, A. Clerk, and K. Schwab, Nature 463, 72 (2010).

[23] S. Vyatchanin, Dokl. Akad. Nauk SSSR 234, 1295 (1977).

[24] S. Mancini, D. Vitali, and P. Tombesi, Phys. Rev. Lett. 80, 688 (1998). 
[25] F. Marquardt, J. P. Chen, A. A. Clerk, and S. M. Girvin, Phys. Rev. Lett. 99, 093902 (2007).

[26] I. Wilson-Rae, N. Nooshi, W. Zwerger, and T. J. Kippenberg, Phys. Rev. Lett. 99, 093901 (2007).

[27] C. Genes, D. Vitali, P. Tombesi, S. Gigan, and M. Aspelmeyer, Phys. Rev. A 77, 033804 (2008).

[28] S. Danilishin, H. Müller-Ebhardt, H. Rehbein, K. Somiya, R. Schnabel, K. Danzmann, T. Corbitt, C. Wipf, N. Mavalvala, and Y. Chen, e-print arXiv:0809.2024 [quant-ph].

[29] A. D. O'Connell, M. Hofheinz, M. Ansmann, R. C. Bialczak, M. Lenander, E. Lucero, M. Neeley, D. Sank, H. Wang, M. Weides, J. Wenner, John M. Martinis, and A. N. Cleland, Nature 464, 697 (2010).

[30] V. B. Braginsky and F. Ya. Khalili, Quantum Measurement (Cambridge Univ. Press, Cambridge, 1992).

[31] D. Vitali, S. Gigan, A. Ferreira, H. R. Böhm, P. Tombesi, A. Guerreiro, V. Vedral, A. Zeilinger, and M. Aspelmeyer, Phys. Rev. Lett. 98, 030405 (2007).

[32] C. Genes, D. Vitali, and P. Tombesi, New J. Phys. 10, 095009 (2008).

[33] M. Bhattacharya, P. L. Giscard, and P. Meystre, Phys. Rev. A 77, 030303(R) (2008).

[34] S. Mancini, V. Giovannetti, D. Vitali, and P. Tombesi, Phys. Rev. Lett. 88, 120401 (2002).

[35] M. J. Hartmann and M. B. Plenio, Phys. Rev. Lett. 101, 200503 (2008).
[36] H. Müller-Ebhardt, H. Rehbein, R. Schnabel, K. Danzmann, and Y. Chen, Phys. Rev. Lett. 100, 013601 (2008).

[37] A. Ferreira, A. Guerreiro, and V. Vedral, Phys. Rev. Lett. 96, 060407 (2006).

[38] S. Pirandola, S. Mancini, D. Vitali, and P. Tombesi, Phys. Rev. A 68, 062317 (2003).

[39] A. Peres, Phys. Rev. Lett. 77, 1413 (1996).

[40] M. Horodeckia, P. Horodeckib, and R. Horodecki, Phys. Lett. A 223, 1 (1996).

[41] L. M. Duan, G. Giedke, J. I. Cirac, and P. Zoller, Phys. Rev. Lett. 84, 2722 (2000).

[42] R. Simon, Phys. Rev. Lett. 84, 2726 (2000).

[43] R. F. Werner and M. M. Wolf, Phys. Rev. Lett. 86, 3658 (2001).

[44] G. Vidal and R. F. Werner, Phys. Rev. A 65, 032314 (2002).

[45] A. Serafini, Phys. Rev. Lett. 96, 110402 (2006).

[46] G. Adesso and F. Illuminati, J. Phys. A: Math. Theor. 40, 7821 (2007)

[47] C. W. Gardiner and P. Zoller, Quantum Noise, 3rd ed. (Springer, Berlin, 2004).

[48] H. Miao, S. Danilishin, H. Müller-Ebhardt, and Y. Chen, e-print arXiv:1003.4048.

[49] C. Genes, A. Mari, P. Tombesi, and D. Vitali, Phys. Rev. A 78, 032316 (2008).

[50] H. Miao, S. Danilishin, H. Müller-Ebhardt, H. Rehbein, K. Somiya, and Y. Chen, Phys. Rev. A 81, 012114 (2010).

[51] [http://www.ligo.caltech.edu/advLIGO]. 